\title{
Microwave Curing of Multidirectional Carbon Fiber Reinforced Polymer Composites
}

\author{
Yingguang Lia ${ }^{\mathrm{a}}$, Nanya $\mathrm{Li}^{\mathrm{b}}$, Jing Zhou ${ }^{\mathrm{a}}$, Qiang Cheng ${ }^{\mathrm{c}}$
}

a College of Mechanical and Electrical Engineering, Nanjing University of Aeronautics and Astronautics, Nanjing, 210016, China

b Institute for Pulsed Power and Microwave Technology, Karlsruher Institute of Technology, Eggenstein-Leopoldshafen, 76344, Germany

c State Key Laboratory of Millimeter Waves, Department of Radio Engineering, Southeast University, Nanjing 210096, China

\begin{abstract}
Composite microwave curing technologies have been researched and given great expectations for decades to cut down the long curing cycle and enormous energy consumption during traditional curing process. However, this good vision was stopped since microwave cannot penetrate and heat multidirectional carbon fiber reinforced polymer composites. In this paper, the mechanism that multidirectional composite cannot be heated was revealed. New approach that the metal strips can stimulate Vertical Penetrating Microwave (VPM) to heat multidirectional carbon fiber reinforced polymer composites was founded. The theory and model of VPM's heating depth of multidirectional composite were established and verified. The results indicated that $2.3 \mathrm{~mm}$ thickness multidirectional composite can be cured effectively by using the VPM.
\end{abstract}

Key words: Directly microwave heating; Vertical penetrating microwave;

Multidirectional carbon fiber; Fiber reinforced polymer composites.

\footnotetext{
*Corresponding author: Professor Yingguang Li

College of Mechanical and Electrical Engineering, Nanjing University of Aeronautics and Astronautics, Nanjing, 210016, China. Tel.: +86 25 84895835; fax: +86 2584895906 .

E-mail address: liyingguang@nuaa.edu.cn
} 


\section{Introduction}

Nowadays, large amounts of carbon fiber reinforced composite parts (CFRP) have been used in the aerospace field without controversy, as the high strength, low density and long fatigue life in strict environment [1]. The traditional autoclave curing technology manufactured large amount of composite parts, because of its well adaptability for composite parts with various shape, simple preparation process and good repeatability. However, the extremely long curing cycle and high energy consumption restrict its further development. For example, the annual output target of A350XWB long haul passenger aircraft (53\% composite usage of total weight) is 156 after 2018, thus 312 composite wing parts should be manufactured. The curing period for one composite part may exceed 24 hours, and the energy consumption is beyond $5000 \mathrm{KW}$ per hour for a $5 \mathrm{~m}$ diameter, $25 \mathrm{~m}$ long autoclave. If half of the curing period and energy consumption can be reduced, the annual output may be double and cost for each aircraft should be reduced.

In recent years, extensive work has been conducted on the quick and energy saving microwave curing technologies of composite materials. Link et al. [2] exhibited the energy saving and fast processing advantages of microwave for heating the glass and carbon fiber reinforce polymer composites. Chaowasakoo and Sombatsompop [3] also showed that the microwave cured composites consumed shorter cure time and had higher impact strength than the conventional thermal cured one. Thostenson and Chou [4] discussed the of microwave heating for composite materials and considered that the 
application of microwave for processing polymer composite materials had a more efficient heat transfer over traditional heat transfer method. Boey and Yap [5] reported that the microwave curing was more effective than thermal heating in accelerating the reaction rates of matrix resin. Zhou et al. [6] demonstrated that microwave reduced the bonding time of polymer reisn compared with thermal proces, which resulted from faster curing of the adhesives. Kayser et al. [7] researched a continuous pultrusion system for carbon fiber reinforced composite materials and the fast and volumetric heating of microwave lead to higher energy efficiency and higher production speed. For the mechanical properties of microwave cured composites, Kwak et al. [8] studied the mechanical properties of composite laminates cured by the VHM (Vötsch Hephaistos Microwave) microwave oven, which can provide a homogeneous electromagnetic field distribution. The microwave cured composite showed higher mechanical performance than the thermal cured one. Joshi and Bhudolia [9] studied the combination of microwave and thermal curing process, and the mechanical properties in tension and flexure of carbon fiber reinforced polymer [0/90] and [ \pm 45$]$ laminates cured with this process were about $5 \%$ better than the autoclave cured laminates. Choe et al. [10] reported by using microwave heating method to fabricating low-density phenolic foam and decreased the environmental pollution. Other research results demonstrated by Li et al. [11] showed that the microwave curing technologies could reduce the cure-induced strain of composite materials. Zhou et al. [12] studied the compensating method of the uneven temperature distribution of composite materials during microwave heating 
process. The experimental results showed improvement in temperature uniformity compared with existing microwave heating methods. Li et al. [13] measured the temperature of composite materials along the thickness direction during microwave curing and demonstrated that the uneven temperature distribution of composites in thickness direction for large-thick composite materials. Ramopoulos et al. [14] developed a system for in-situ monitoring of curing processes of thermoset resins under microwave heating. The dielectric properties of polymer resin at different temperature was measured.

However, the microwave cannot directly heat the multidirectional carbon fiber reinforced polymer composites stopped the development of microwave heating technologies in aerospace filed. In 1984, Lee and Springer [15] researched the microwave curing technology of unidirectional and multidirectional carbon fiber reinforced epoxy composites. They reported that the microwave reflectance was very high (about 100\%) for multidirectional carbon fiber composites, and microwave cannot even heat two layers of multidirectional composite. Paulauskas [16] from the OAK Ridge National Laboratory and Lockheed Martin issued a report and indicated that variable frequency microwave does not work for the multidirectional carbon fiber laminates. Thostenson and Chou [4] demonstrated that the application of microwave heating to multidirectional carbon fiber composites may be limited as the high conductivity of carbon fibers. Kaiser [17] from Deutsche Aerospace measured the absorption of microwave from $2 \mathrm{GHz}$ to $100 \mathrm{GHz}$ in multidirectional carbon fiber 
reinforced composite. The microwave absorption of multidirectional composite can be increased in high frequency, but only the first composite layer with carbon fiber direction perpendicular to the electric field direction have attribution to the absorption. Until now, Kwak et al. [18] reported that the microwave still can only cure unidirectional carbon fiber reinforced polymer composite, but no substantive progress for multidirectional carbon fiber reinforced polymer composites. Feher [19] found a compromised approach to indirectly heat the multidirectional composite by using microwave absorbing film.

This problem has perplexed researchers from the day that microwave was attempt to apply in composite heating process. In this paper, a new microwave heating method by stimulating Vertical Penetrating Microwave (VPM) using metal strip array is presented to directly heat multidirectional carbon fiber reinforced composite. The heating depth of multidirectional composite under the radiation of VPM was calculated and verified.

\section{Experiments}

\subsection{Materials and devices used}

The composite materials used in this experiment are T700/QY9611 continuous carbon fiber reinforced bismaleimide prepreg with the matrix volume fraction of $35.7 \%$. This kind of prepreg has high usage temperature and strength, usually applied in primary load bearing composites of high-speed fighter planes. Other assisted materials are supplied by Airtech Company, such as high temperature resistance vacuum bag VB- 
3, release film SK2RF230-1BL (0.02mm thickness) and so on. The fiber Bragg grating sensors (MOI company, $0.15 \mathrm{~mm}$ diameter) were applied to measure the temperature distribution of composite samples at different thickness. The MOI SM125 fiber Bragg grating demodulator instrument and octagon microwave heating system were employed. The tempered glass tooling and copper strips $(0.01 \mathrm{~mm}$ thickness $)$ were utilized. In order to avoid arcing of the carbon fibers, the aluminum tape should be used to stick on the edge of composite samples.

\subsection{Measurement of transmissivity}

In order to study the microwave heating mechanism of unidirectional carbon fiber reinforced polymer composites and the reason that multidirectional carbon fiber reinforced polymer composites cannot be heated by microwave energy, the microwave transmissivity of composite samples were measured. Two different electric field direction were applied, one is parallel to the fiber axial direction of the first laminate and another is vertical to the fiber axial direction (longitudinal direction). Both of the propagation direction of those two microwave modes are normal to the surface of composite samples. The size of testing samples were $200 \mathrm{~mm} \times 200 \mathrm{~mm}$, and the thickness is $1 \mathrm{~mm}$ and $3 \mathrm{~mm}$. The schematic of transmissivity measurement of composite samples is shown in Fig. 1. The transmitting and receiving antennas are placed on the opposite side of the sample and the focusing lens are installed to reduce the deviation of measurements. Traveling microwave range from $1 \mathrm{GHz}$ to $8 \mathrm{GHz}$ can be generated and received by using the Agilent microwave network analyzer. The T700/QY9611 carbon 
fiber reinforced bismaleimide composite material was used to prepare the test samples. The composite samples for microwave transmissivity testing are shown in Table 1. U_P and M_P samples are unidirectional and multidirectional carbon fiber reinforced bismaleimide composite respectively, and the electric field direction is parallel to the longitudinal direction of carbon fiber.

\subsection{Measurement of heating rate and heating depth of composites}

In order to obtain the highest heating efficiency of VPM, the heating rate of composite materials should be tested when applying different parameters of metal strips and insolation material. The heating rates of multidirectional CFRP were measured by using Fiber Bragg Grating (FBG) sensors, which buried in the composite samples. The influence of cure-induced strains was avoided by packaging the FBG sensor with a glass capillary tube. Base on the stimulation theory of vertical penetrating microwave described in section 3.3, three important factors were considered in this experiments. As shown in Table 2, the width of copper strip, width between copper strips and the height of insulation material are designed and tested. The parameters in the table are acquired according to the previous experiments and coplanar waveguide theory. Because of the copper material has good electric conductivity, the copper strips were applied in this experiments. And the insulation material can be release film (SK2RF230-1BL) used in vacuum bagging process. The size of test sample is $200 \mathrm{~mm} \times 200 \mathrm{~mm} \times 3.5 \mathrm{~mm}$ and its ply sequence is $\left[0^{\circ} / 45^{\circ} / 90 \% / 0 \%-45 \% / 0 / 90 \% / 45 \%{ }^{\circ}\right]_{s}$. The axial direction of carbon fiber in the first layer of the samples shown in the table are parallel to the longitudinal 
direction of copper strips. Other samples named from V_A to V_E had the same testing parameters with $\mathrm{P}_{-}$A to $\mathrm{P}_{-} \mathrm{E}$, but the axial direction of carbon fiber in the first layer were vertical to the longitudinal direction of copper strips. The $1000 \mathrm{~W}$ microwave power was applied into the octagon microwave chamber during the experiments.

For the sake of calculating the heating depth of vertical penetrating microwave, the microwave energy absorbed by material at different thickness should be calculated. In the case of non-magnetic materials, the absorbed microwave energy per unit volume of composite samples $\left(Q^{m}\right)$ can be calculated as:

$$
Q^{m}=2 \pi f \varepsilon_{0} \varepsilon^{\prime \prime} E^{2}
$$

where $\varepsilon_{0}$ is the dielectric property of air, $\varepsilon^{\prime \prime}$ is the dielectric loss of the composite, $f$ is the frequency of microwave, and $E$ is the electric field intensity. In this paper, the heating depth is defined as the depth at which the electric field magnitude of the microwave decreases to $1 / 2 e(18.4 \%)$ of its original magnitude. Thus, the heating depth of the multidirectional CFRP can be calculated as below:

$$
E_{\text {ratio }}=\frac{E_{z=D_{t}}}{E_{z=0}}=\sqrt{\frac{Q_{z=D_{t}}^{m}}{Q_{z=0}^{m}}}=\frac{1}{2 e}
$$

Where, $E_{\text {ratio }}$ is the ratio of electric field intensity between total intensity ( $\left.E_{z=0}\right)$ and the electric field intensity $\left(E_{z=D_{t}}\right)$ at depth $D_{t}$. The total microwave energy is $Q_{z=0}^{m}$, and the microwave energy at depth $D_{t}$ is $Q_{z=D_{t}}^{m}$. However, the electric field intensity and absorbed microwave energy in the composite materials cannot be measured directly.

The calculation method of heating depth base on the heating rate of different thickness is shown in Fig.2. In order to improve the accuracy, three samples were tested 
for each measurement. The heat quantity on surface of composite samples can be defined as $Q_{z=0}$ (or $\left.Q_{z=0}^{\prime}\right)$, and the heat quantity at depth $D_{t}$ of composite samples can be defined as $Q_{z=D_{t}}$ (or $Q_{z=D_{t}}^{\prime}$ ). The heat transfer of samples during VPM heating process is shown in Fig.2(a). Obviously, the heat quality at a certain depth contains two parts, which are the microwave heating and the heat transfer in the materials. Because the temperate raising rate at a certain depth can be measured. Thus, by excluding the heat transfer in composite material, the microwave energy absorbed by the material can be calculated.

As shown in Fig. 2(b), the composite samples heated by using conventional heating platform was designed to has the same the heat transfer direction with VPM heating process. Note that the heat transfer to the ceramic tool can be ignored since its thermal conductivity is very small compared with the composite. For any small elements in the composite material, the Fourier heat transfer law can be used to relate the electric field intensity and temperature rising rate. Hence, the heat quality of composite materials that generate by absorbing microwave energy can be calculated as below.

$$
\begin{aligned}
& Q_{z=0}^{m}=Q_{z=0}^{\prime} \\
& Q_{z=D_{t}}^{m}=Q_{z=D_{t}}-Q_{z=D_{t}}^{\prime}
\end{aligned}
$$

Assuming the VPM heating and conventional thermal heat transfer have the same heat quality at the surface of composite materials $\left(Q_{z=0}^{\prime}=Q_{z=0}\right)$. The transient heat quality of composite samples generated by absorbing microwave at a certain depth can be calculated easily according to equation (4). 


\section{Results and discussions}

\subsection{Microwave heating mechanism of unidirectional CFRP}

The experimental results of microwave transmissivity of different composite samples are shown in Fig. 3. Several groups of samples were tested and showed almost the same results. For the purpose of easy to discussion, one group of the testing results are exhibited. It's obvious that unidirectional carbon fiber reinforced composite has the highest transmittance of the microwave which vertical to the longitudinal direction of carbon fiber. At the frequency range from $2 \mathrm{GHz}$ to $3 \mathrm{GHz}$, about $12 \%(9.2 \mathrm{~dB})$ travelling microwave still can penetrate the samples and received by the antenna. Because of the very low reflection value detected by the analyzer, above $80 \%$ microwave energy is absorbed by the composite material. For the U_P_1 sample, the microwave nearly cannot penetrate the composite material, and very high reflection is obtained. After the thickness of unidirectional samples increase to $3 \mathrm{~mm}$, the difference of transmissivity between $U_{-} P_{-} 3$ and $U_{-} V_{-} 3$ can only be found at high frequency. However, no matter the direction of electric field is parallel or vertical to the longitudinal direction of carbon fiber, the microwave always cannot penetrate multidirectional carbon fiber reinforced composite, even for $1 \mathrm{~mm}$. The reason will be explained in section 3.2 .

For the unidirectional CFRP, carbon fiber bundles are aligned to one direction and polymer resin is distributed among the carbon fibers. The electric conductivity along the length direction of carbon fiber is about $10^{5} \mathrm{~S} / \mathrm{m}$, but the transverse and thickness 
direction is about $10^{2} \mathrm{~S} / \mathrm{m}$. Because of the different conductivity in three directions of composite, the anisotropic electromagnetic properties of unidirectional CFRP have different scattering characteristics to the incident microwave, shown in Fig.4.

As illustrates in Fig.4 (a), almost all of the microwave energy is reflected and cannot transmit into the composite when the polarization direction of electric field is parallel to longitudinal direction of carbon fiber. The induced strong conducting current will result in the intense scattering waves that destructively interference with the incident waves and thus leading to nearly total reflection at the composite interface. On the contrary, when the longitudinal direction of carbon fiber perpendicular to the polarization direction, nearly all of the microwave energy can be transmitted through composite, as shown in Fig.4 (b). In this case, the induced current is very limited in the transverse direction, which has little influence on the transmission of electromagnetic waves. Therefore, large microwave transmissivity can be observed in the experiment.

In the microwave resonant cavity, the cavity mode can be decomposed into plane waves from different directions with various polarization directions. The wave polarized parallel to the carbon fiber will be reflected. However, the wave polarized perpendicular to the carbon fiber can directly heat the composite. Under such circumstances, unidirectional CFRP can be heated in the microwave cavity.

\subsection{Microwave non-heating mechanism of multidirectional CFRP}

The electric filed direction of the microwave in the cavity can be divided into three directions, as shown in Fig. 5. Where, u component is in the direction along the carbon 
fiber of first layer, v component is vertical to the carbon fiber of first layer, and w component is along the normal direction of the composite material surface. The plane determined by the $\mathrm{u}$ and $\mathrm{v}$ components is parallel to the surface of the composite. For these two components, $\mathrm{u}$ component will be reflected by the first layer of CFRP. Even the $\mathrm{v}$ component can transmit through the first layer, it will also be reflected by the subsequent layers. Due to the incident microwave will be counteracted by the reflected microwave based on the transmission matrix theory. The whole multidirectional CFRP exhibits zero electric potential and totally reflect $u$ and $v$ components.

With regards to the w component, its electric field direction is vertical to the composite surface, and its transmission properties will not be affected by the ply direction of CFRP. However, it is well known that the microwave energy is transmit along the propagation direction, and very little energy can be dissipated to the surrounding materials. Therefore, the w component cannot heat multidirectional CFRP as well, because the microwave energy propagates outside the composite material. The infrared thermal images (FLIR A300, more detail can be found in Zhou et al. 2018) of unidirectional and multidirectional carbon fiber reinforced bismaleimide composite parts $(200 \times 200 \mathrm{~mm})$ which placed in the microwave cavity are shown in Fig. 6 . Apparently, the unidirectional CFRP can be heated effectively, but the temperature of the multidirectional CFRP remains unchanged. This consequence coincides with the transmissivity testing results in Fig. 3. 


\subsection{Multidirectional CFRP heated by using Vertical Penetrating Microwave (VPM)}

\subsubsection{Definition of the Vertical Penetrating Microwave}

According to the above analysis, the Vertical Penetrating Microwave (VPM) is proposed to heat multidirectional CFRP, which is defined as the polarization direction of electric field along the normal direction of the composite surface and the microwave energy propagates inside the composite material. Therefore, the microwave energy can penetrate the multidirectional CFRP and be absorbed by the material.

\subsubsection{VPM generated by using metal strips in microwave cavity}

In order to generate the VPM in composite material, the coplanar waveguide structure was studied by reference. The coplanar waveguide has an electric field vertical to the dielectric material that cover by the metal strips on the top and bottom surface. Therefore, metal strip structures are designed and shown in Fig. 7. The metal strips are placed on the tool surface and an insolation film is used to separate the metal strip array and the CFRP. The metal strips are applied on the top and bottom surface of CFRP, as shown in the cross-section of Fig.7. According to the coplanar waveguide theory, the metal strips and multidirectional CFRP can be equivalent to the conductor and dielectric materials, respectively.

For the metal strip structure mentioned above, the experiment results of heating rate of different structure parameters are shown in Fig. 8. Because the temperature distribution of composite is not uniform, so the highest heating rate of each sample are calculated. Four samples for each parameter setting are tested and the standard 
deviations are also shown in Fig. 8. When the axial direction of carbon fiber in the first layer parallel to the longitudinal direction of copper strips, which illustrated in Fig.8 (a), the highest heating rate can reach $27^{\circ} \mathrm{C} / \mathrm{min}$. This is owing to the reason that the largest induced currents in the longitudinal direction of copper strips can be stimulated. For both Fig. 8 (a) and (b), by decreasing the height of insulation material (d value), the heating rate increased rapidly. That means reducing the distance between the composite and metal strips can enhance the electric field intensity of VPM. According to the experiment results, $\mathrm{P}_{-} \mathrm{B}$ sample has the highest heating efficiency. Thus, the subsequent experiments will apply this parameter: the height of insulation film is $0.02 \mathrm{~mm}$, and the copper strips have a width of $8 \mathrm{~mm}$, with a space of $13 \mathrm{~mm}$ between any two of them.

On account of the copper strip is only about $0.01 \mathrm{~mm}$, thus the heat induced by skin effect should be tested to eliminating the possibility that the VPM heat the strips rather than composite material. Through placing T1 FBG sensor on the composite surface, and a FBG sensor stick on the copper strip (marked as Strip in Fig. 9) which placed under T1 point, the temperature curves are measured. And the T2 and T3 FBG sensors are randomly placed on the composite surface. The measuring result of one sample exhibits in Fig. 9. It is obvious that the temperature of copper strip is much lower than the composite material. At the peak of temperature curve, the composite sample arrives at $65^{\circ} \mathrm{C}$, but the copper strip is only $38^{\circ} \mathrm{C}$. As a conclusion, the vertical penetrating microwave can directly heat the multidirectional carbon fiber reinforced composite material. 
The infrared thermal image during the heating rate measurement and the simulation result of multidirectional CFRP are shown in Fig. 10. The Comsol Multiphysics simulation software is utilized to calculate the temperature distribution of composite material with the copper strip array covered. The homogeneous material model is used and the dielectric property of composite is 40 (the value is obtained by equivalent this situation to that the electric field direction is vertical to the longitudinal direction of carbon fiber). Other material parameters including copper and insolation film are directly form the material library of Comsol Multiphysics. The temperature distribution pattern of measured result is consistent with the simulation outcome. Standing wave is established between composite and copper strips. It can be concluded that the copper strip array is in role of microwave antenna and ground plate of resonant coplanar waveguide. The VPM stimulated by the copper strip array and composite structure can be strongly absorbed by the multidirectional CFRP itself.

\subsubsection{Heating depth model of multidirectional CFRP by using VPM}

The electric field generated by metal strips have two different modes, one is odd mode which shown in Fig. 11 (a) and the other is even mode as shown in Fig. 11 (b). Those two different resonant mode significantly affected the intensity and distribution of the electric field. The more electric field lines along the normal direction of the composite surface in, the better the heating efficiency will be. Obviously, the odd mode need to be encouraged, and the even mode should be restricted. 
According to the coplanar waveguide theory, the intensity and distribution of the electric field of the VPM in the multidirectional CFRP can be calculated. As shown in Fig. 11 (c), the coordinate origin is set on the center of the metal strip, the thickness of CFRP material is $h$, the width of metal strip is $S$, the space between metal strips is $g$. The dielectric properties of air and the CFRP material are $\varepsilon_{0}$ and $\varepsilon_{r}$. The top view of the strip-CFRP structure is illustrated in Fig. $11(\mathrm{~d})$. Assuming that the electric walls are placed in the coordinate of $X=0$ and $X=\lambda_{g} / 2$, the magnetic wall is placed in the coordinate of $\mathrm{Y}=\mathrm{b} . \lambda_{g}$ is the resonant wavelength of the strips-CFRP structure. The electric field intensity and distribution in the $\mathrm{z}$ direction can be calculated as follows (Simons, 2002):

$$
\begin{aligned}
& E_{z}=-\frac{2 V_{0}}{b} \times \sum_{n>0}^{\infty} \frac{1}{F_{n 1}}\left[\frac{\sin n \pi \chi / 2}{n \pi \chi / 2} \times \sin n \pi \chi^{\prime} / 2\right] \times \cos \frac{n \pi y}{b} \times \\
& \left(\sinh \gamma_{n 1} z-\tanh r_{n} \times \cosh \gamma_{n 1} z\right)
\end{aligned}
$$

Where $z$ is the depth of the CFRP in the thickness direction, $E_{z}$ is the electric field intensity of VPM in Z direction, $V_{0}$ is the initial voltage between two strips, and other variables in the above equation is listed below:

$$
\begin{aligned}
& \chi=\frac{g}{b}, \quad \chi^{\prime}=1 \\
& F_{n 1}=\frac{b \gamma_{n 1}}{n \pi}=\sqrt{1-\left(\frac{2 b u}{n \lambda}\right)^{2}} \\
& u=\sqrt{\varepsilon_{r}-\left(\frac{\lambda}{\lambda_{g}}\right)^{2}} \\
& r_{n}=\gamma_{n 1} h+\tanh ^{-1}\left(\frac{F_{n 1}}{\varepsilon_{r} F_{n}}\right)
\end{aligned}
$$


The $\lambda_{g}$ is the resonant wavelength of the strips-CFRP structure and can be expressed as:

$$
\lambda_{g}=\frac{\lambda}{\sqrt{\varepsilon_{e f f}}}
$$

Base on the coplanar waveguide theory presented by Simons [20], the $\varepsilon_{\text {eff }}$ is the effective dielectric property of the strips-CFRP structure and can be expressed as:

$$
\varepsilon_{\text {eff }}=\frac{1}{2}\left[\varepsilon_{r}+1+\left(\varepsilon_{r}-1\right)\left(1+\frac{10 h}{S}\right)^{-0.5}\right]
$$

Therefore, the parameters related with heating depth of VPM are shown in the equation below:

$$
D_{t}=f\left(R_{s}, S, g, \varepsilon_{r}, h, P, \lambda\right)
$$

Where $R_{s}$ is the resistance of metal strip, $S$ is the width of metal strip, $g$ is the space between metal strips, $h$ is the thickness of CFRP, $\varepsilon_{r}$ is the dielectric property of CFRP, $P$ is the ply direction of CFRP's first layer, $\lambda$ is the wavelength of microwave. The ply direction of first layer that not parallel to the longitudinal direction of metal strips should be avoid. In this paper, the heating depth of the VPM is defined as the depth that electric intensity attenuated to $\frac{1}{2 e}(18.4 \%)$ of the total incident intensity, as shown in equation (2). Base on the equation (5) to (11), the heating depth of the multidirectional carbon fiber reinforced bismaleimide composite under the radiation of 2.45 GHz microwave was calculated. Considering the energy consumption, coupling affection of the adjacent strips and the microwave reflection of copper strips, a coupling coefficient was introduced to the calculation model. On account of the experience data 
and simulation results, the coupling coefficient around 0.35 is appropriate. The calculated result indicated that the VPM $(2.45 \mathrm{GHz})$ heating depth of multidirectional CFRP by using both top and bottom copper strips can achieve $2.3 \mathrm{~mm}$.

For the purpose of measuring the heating depth of the multidirectional composite materials, FBG sensors were buried in different depths of the test sample to monitor the temperature raising rate. The average heating rates and ratios of electric field intensity at different thicknesses of three samples are summarized in Table 3. As shown, at least $1.13 \mathrm{~mm}$ thickness multidirectional CFRP composite can be penetrated and heated by using the copper strip array at the bottom of the composite material. Thus, the heating depth can be improved to about $2.3 \mathrm{~mm}$ by applying metal strips at the top and bottom surface of the multidirectional CFRP, which verified the accuracy of the heating depth model. Further improvement of the heating depth can be realized by designing the parameters of the metal strip array reasonably, which is to be conducted in the authors' ongoing research work.

Finally, one typical part of multidirectional carbon fiber reinforced bismaleimide composite (length and width: $200 \times 200 \mathrm{~mm}$ ) with $2.3 \mathrm{~mm}$ thickness is manufactured by using the VPM. The preparation process is shown in Fig. 12 (a) and the final cured multidirectional CFRP is shown in Fig.12 (b). The surface temperature distribution of the composite material is shown in Fig. 12(c) and (d). Fig. 12(c) is captured without vacuum bag, and the shadow in Fig. 12(d) is the drape of vacuum bag. It can be seen that the multidirectional CFRP is directly heated by the stimulated VPM. The CFRP at 
the copper strips area has a relative higher temperature than the area on the space between copper strips. This is because the electric field lines of the VPM are concentrated at the surface of the copper strips, which leads to a higher electric field intensity compared with the space between them. The curing temperature measured by FBG sensors are shown in Fig. 13. Two of them buried in the middle and top surface of composite material. The temperature difference occurred at the dwell stage of $200^{\circ} \mathrm{C}$, and no obvious reaction exothermic phenomenon is founded. A quick post curing process is applied before the cooling down process. The testing results of differential scanning calorimetry exhibit that the composite part was totally cured.

\section{Conclusion}

In this work, the VPM was proposed and stimulated by using the metal strip array to effectively heat multidirectional CFRPs. The heating mechanism of unidirectional CFRPs and non-heating mechanism of multidirectional CFRPs by microwave were revealed. Based on the coplanar waveguide theory, the calculation model of the heating depth of the VPM with the parameters of metal strips, composite material and microwave frequency was established and verified. Experimental results indicated that the heating depth of the VPM is about $2.3 \mathrm{~mm}$ by applying metal strips at the top and bottom surface of the multidirectional composite. Further improvement of the heating depth can be realized by designing the parameters of the metal strip array reasonably, which is to be conducted in the authors' ongoing research work. 


\section{Acknowledgements}

This project was supported by National Natural Science Foundation of China (Grant no. 51575275).

\section{Reference}

[1] Li Y, Li N, Gao J. Tooling design and microwave curing technologies for the manufacturing of fiber-reinforced polymer composites in aerospace applications. Int. J. Adv. Manuf. Technol. 2014; 70(1-4): 591-606.

[2] Link G T, Kayser A, Melcher D, Prastiyanto S, Soldatov J, Jelonnek J. Microwave Materials Processing at the Karlsruhe Institute of Technology. Engineering International Conference “UNNES Conservation” Proceeding. 2013; pp: 4-9.

[3] Chaowasakoo T, Sombatsompop N. Mechanical and morphological properties of fly ash/epoxy composites using conventional thermal and microwave curing methods. Compos. Sci. Technol 2007; 67: 2282-2291.

[4] Thostenson E, Chou T W. Microwave processing: fundamentals and applications. Compos. Part A. 1999; 30: 1055-1071.

[5] Boey F, Yap B. Microwave curing of an epoxy-amine system: effect of curing agent on the glass-transition temperature. Polym. Test. 2001; 3: 837-845.

[6] Zhou S, Hawley M C. A study of microwave reaction rate enhancement effect in adhesive bonding of polymers and composites. Compos. Struct. 2003; 61(4): 303309.

[7] Kayser T, Link G, Seitz T, Nuss V, Dittrich J, Jelonnek J, Heidbrink F, Ghomeshi R. An applicator for microwave assisted pultrusion of carbon fiber reinforced plastic. IEEE MTT-S International Microwave Symposium. 2014; pp: 1-4. 
[8] Kwak M, Robinson P, Bismarck A, Wise R, Middlesbrough U. Curing of composite materials using the recently developed hephaistos microwave. 18th international conference on composite materials. 2011; pp: 21-26.

[9] Joshi S C, Bhudolia S K. Microwave-thermal technique for energy and time efficient curing of carbon fiber reinforced polymer prepreg composites. J. Compos. Mater. 2014; 48(24): 3035-3048.

[10] Choe J, Kim M, Kim J. A microwave foaming method for fabricating glass fiber reinforced phenolic foam. Compos. Struct. 2016; 152: 239-246.

[11] Li N, Li Y, Hao X, Gao J. A comparative experiment for the analysis of microwave and thermal process induced strains of carbon fiber/bismaleimide composite materials. Compos. Sci. Technol. 2015; 106(106): 15-19.

[12] Zhou J, Li Y, Li N, Liu S, Cheng L, Sui S, Gao J. A multi-pattern compensation method to ensure even temperature in composite materials during microwave curing process. Compos. Part A. 2018; 107: 10-20.

[13] Li N, Li Y, Hang X, Gao J. Analysis and optimization of temperature distribution in carbon fiber reinforced composite materials during microwave curing process. J. Mater. Process. Technol. 2014; 214(3): 544-550.

[14] Ramopoulos V, Soldatov S, Link G, Kayser T, Jelonnek J. System for in-situ dielectric and calorimetric measurements during microwave curing of resins. IEEE Microwave Conference. 2015; pp: 29-32

[15] Lee W I, Springer G S. Microwave curing of composites. J. Compos. Mater. 1984; 18: $387-409$.

[16] Paulauskas F L. Variable frequency microwave (VFM) curing, processing of thermoset prepreg laminates. Oak Ridge National Lab 1996; pp: 8-13.

[17] Kaiser J H. Reflection and absorption of microwaves (2-100 GHz) by carbonfiber-reinforced composite surfaces. Journal of Research in Nondestructive Evaluation. 1994; 5(4), 275-283.

[18] Kwak M. Microwave curing of carbon-epoxy composites: process development and material evaluation. Imperial College London. 2016; pp: 28-35. 
[19] Feher L E. Energy efficient microwave systems: materials processing technologies for avionic, mobility and environmental applications. Springer Science Energy. Ecol. Environ 2009; pp:56-78.

[20] Simons R N. Coplanar waveguide circuits, components, and systems, Wiley Series in Microwave and Optical Engineering. 2002; pp:160-165. 


\section{Figure and Table Captions}

Fig.1 Schematic of transmissivity measurement of composite samples.

Fig. 2 Schematics of measuring the VPM's heating depth of multidirectional CFRP, (a)

VPM heating by using copper strips on the bottom of composite sample, (b) heating composite sample by using conventional heating platform.

Fig. 3 Microwave transmissivity of composite samples from $1 \mathrm{GHz}$ to $8 \mathrm{GHz}$, (a) sample U_P_1, (b) sample U_V_1, (c) sample U_P_3, (b) sample U_V_3, (e) sample M_P_1, (f) sample $M_{-} V_{-} 1$.

Fig. 4 Microwave heating mechanism of unidirectional CFRP. (a) the longitudinal direction of carbon fiber is parallel to the polarized direction of electric field, (b) the carbon fiber is vertical to the polarized direction of electric field.

Fig. 5 Mechanism of microwave cannot heat multidirectional CFRP.

Fig. 6 The infrared thermal images of CFRP heated in the microwave cavity. (a) unidirectional CFRP, (b) multidirectional CFRP.

Fig. 7 Vertical penetrating microwave stimulated by metal strips in the cavity.

Fig. 8 Heating rate of different samples by using vertical penetrating microwave.

Fig. 9 Heating curves of composite sample and copper strip.

Fig. 10 Infrared thermal image and simulation result of multidirectional carbon fiber reinforced composite heated by using VPM, (a) infrared thermal image of samples during the heating rate measurement, (b) comsol mutiphysics simulation result.

Fig. 11 Electric field distribution of strips-CFRP structure. (a) odd mode of electric field, (b) even mode of electric field, (c) cross-section of the strips-CFRP structure, (d) top 
view of the strips-CFRP structure.

Fig. 12 (a) Multidirectional CFRP in microwave oven, (b) composite part cured by VPM, (c) infrared thermal image of CFRP at low temperature, (d) infrared thermal image of CFRP at high temperature.

Fig. 13 Curing temperature of multidirectional CFRP heated by VPM.

Table 1. Composite samples for microwave transmissivity testing.

Table 2. Composite samples for the VPM heating rate testing.

Table 3. Heating rates and ratios of the VPM at different thicknesses of the composite samples. 




Microwave network analyzer 


(a)

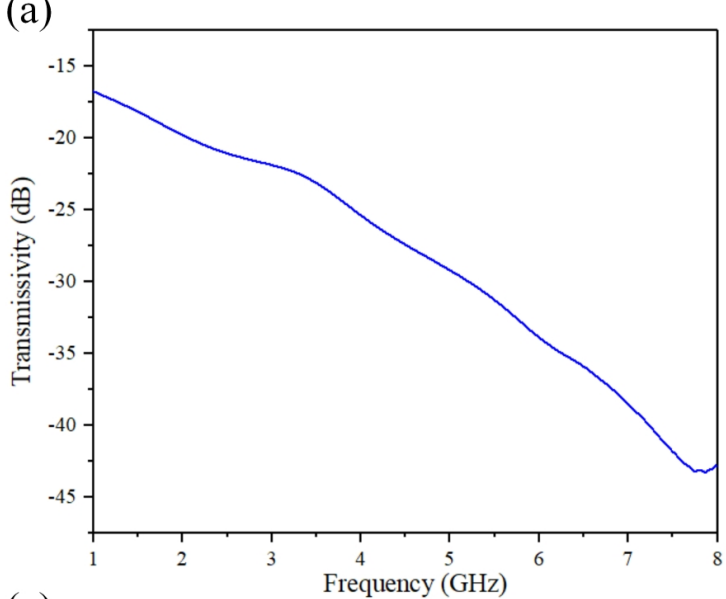

(c)

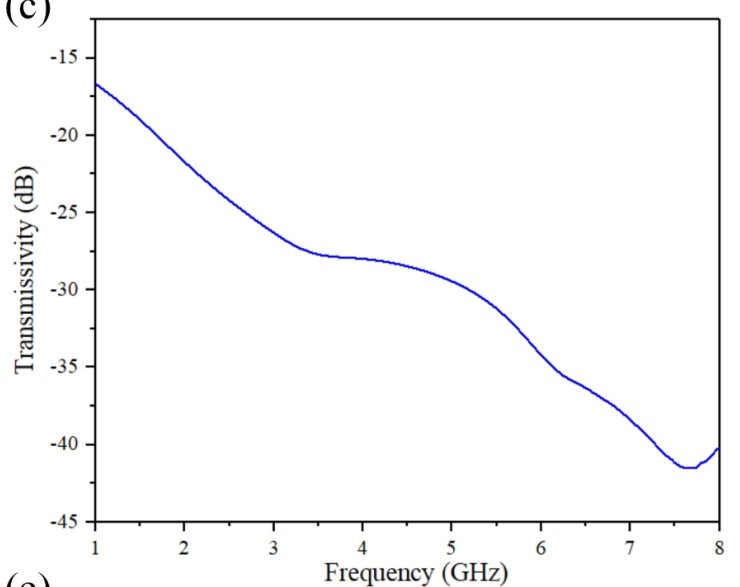

(e)

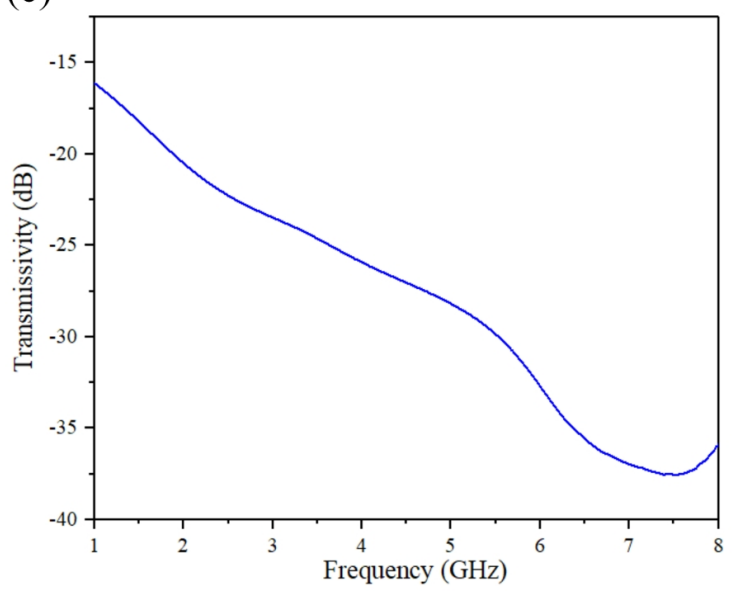

(b)

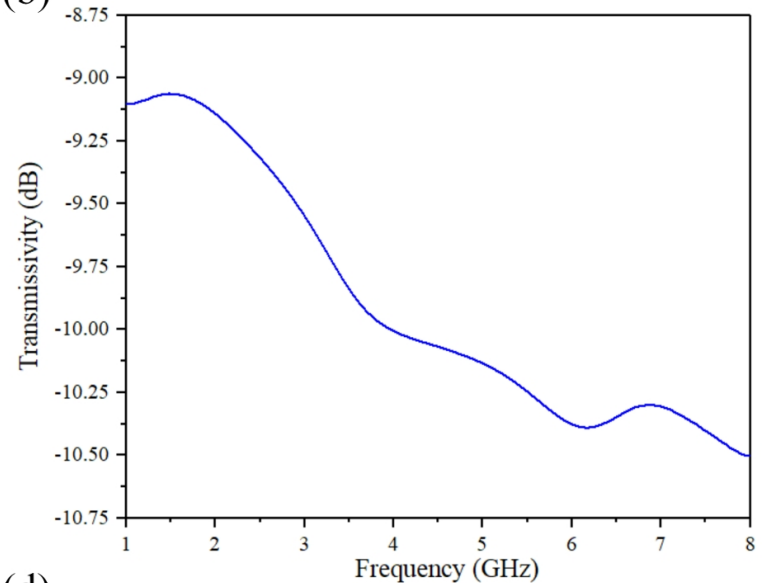

(d)

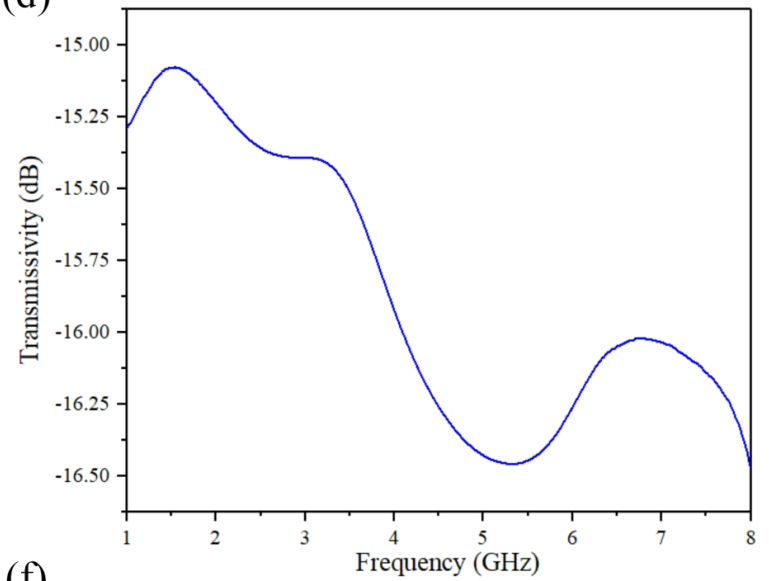

(f)

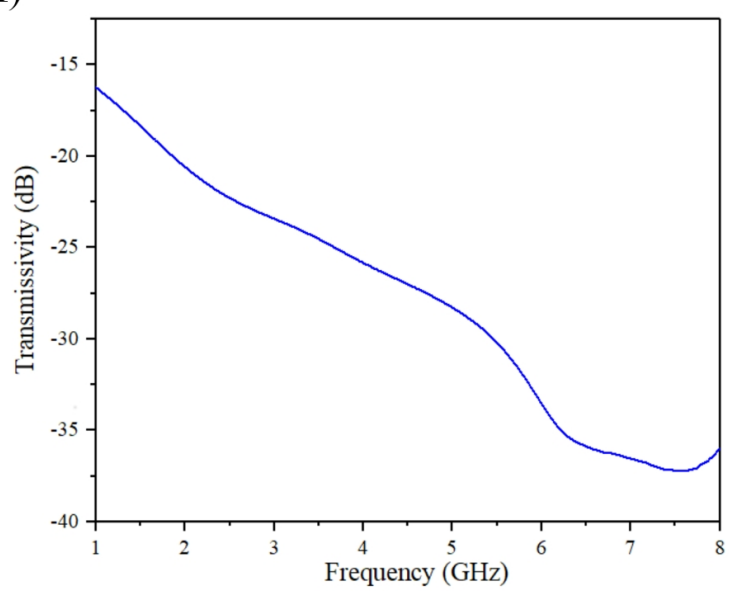


(a)

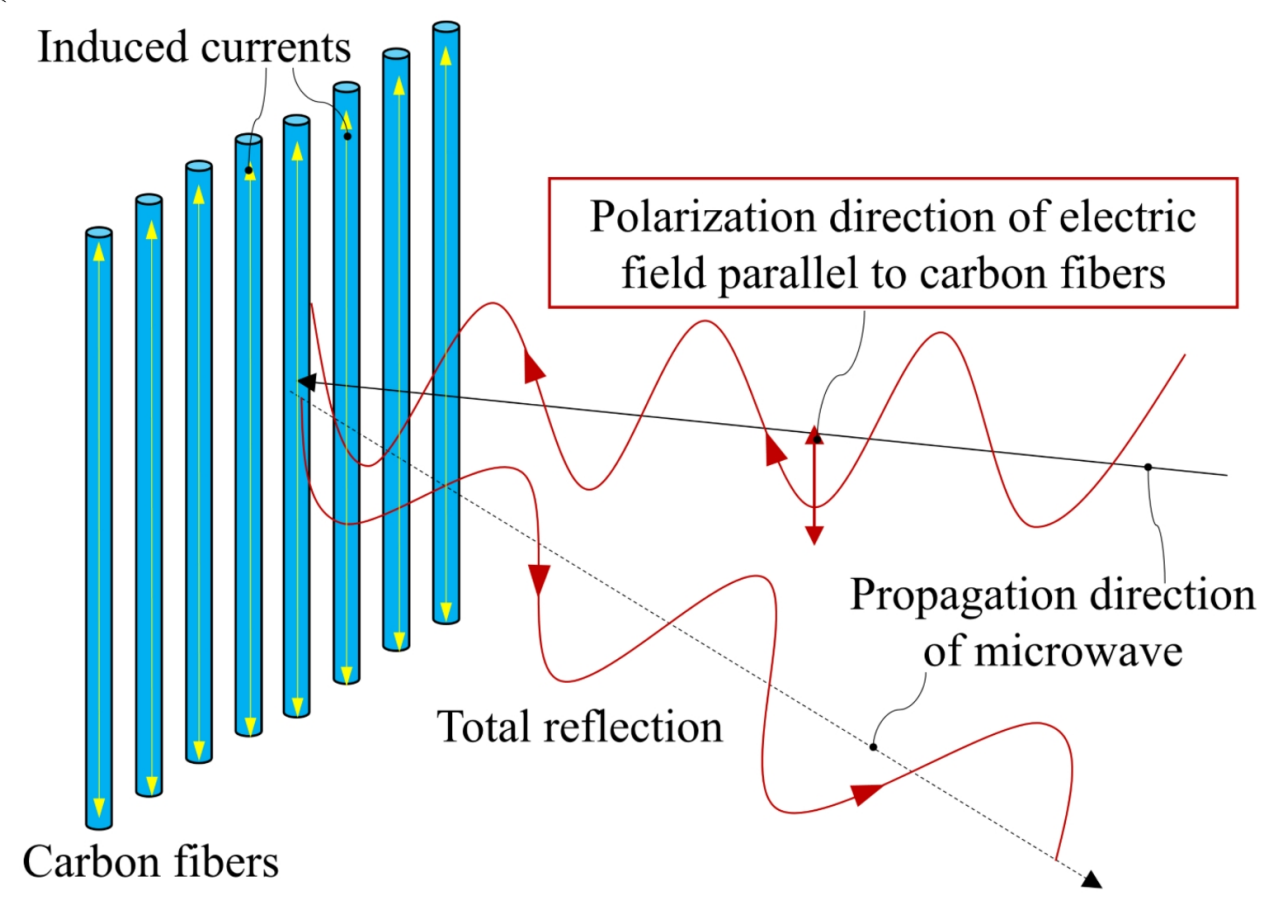

(b)

Transmitted wave

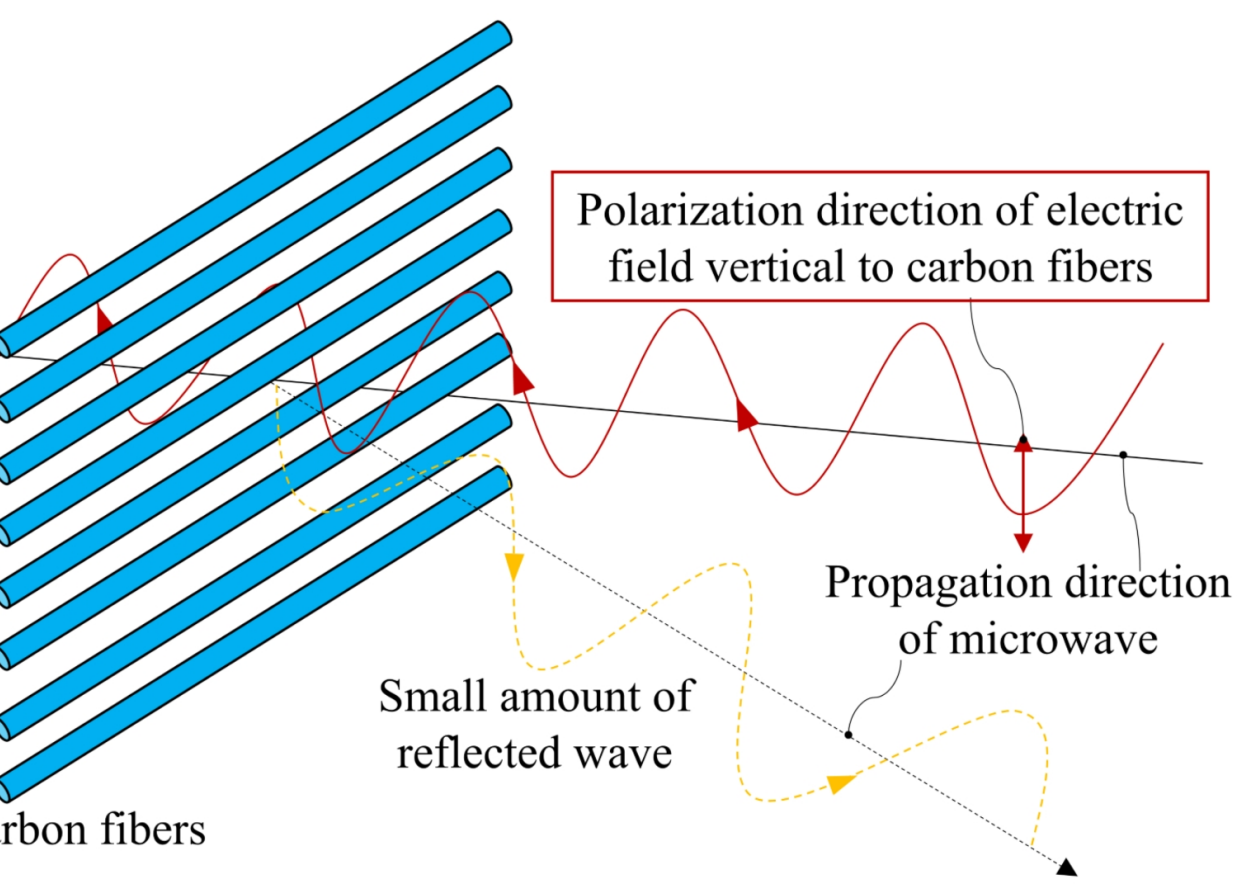




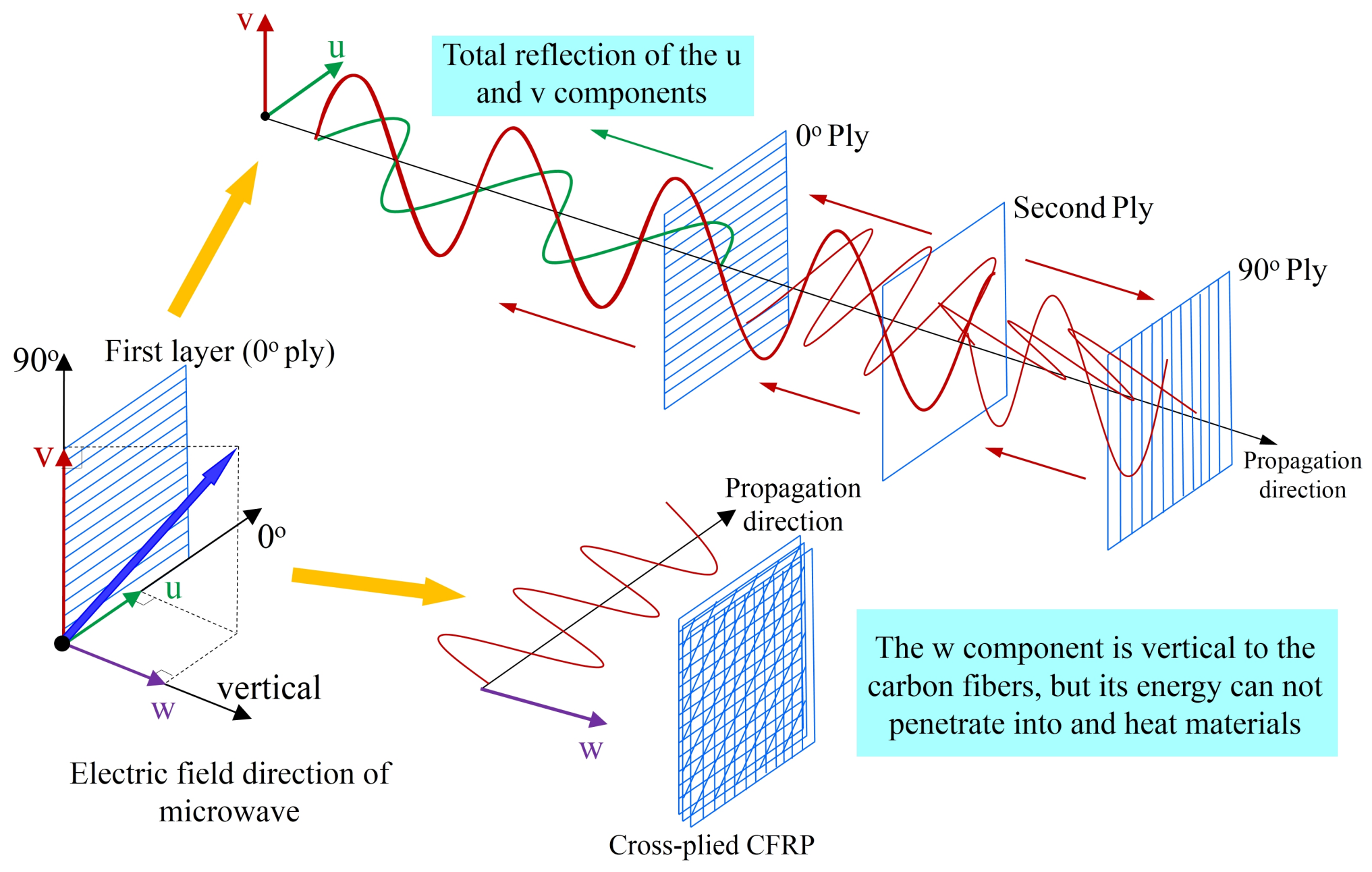


(a)

Unidirectional CFRP

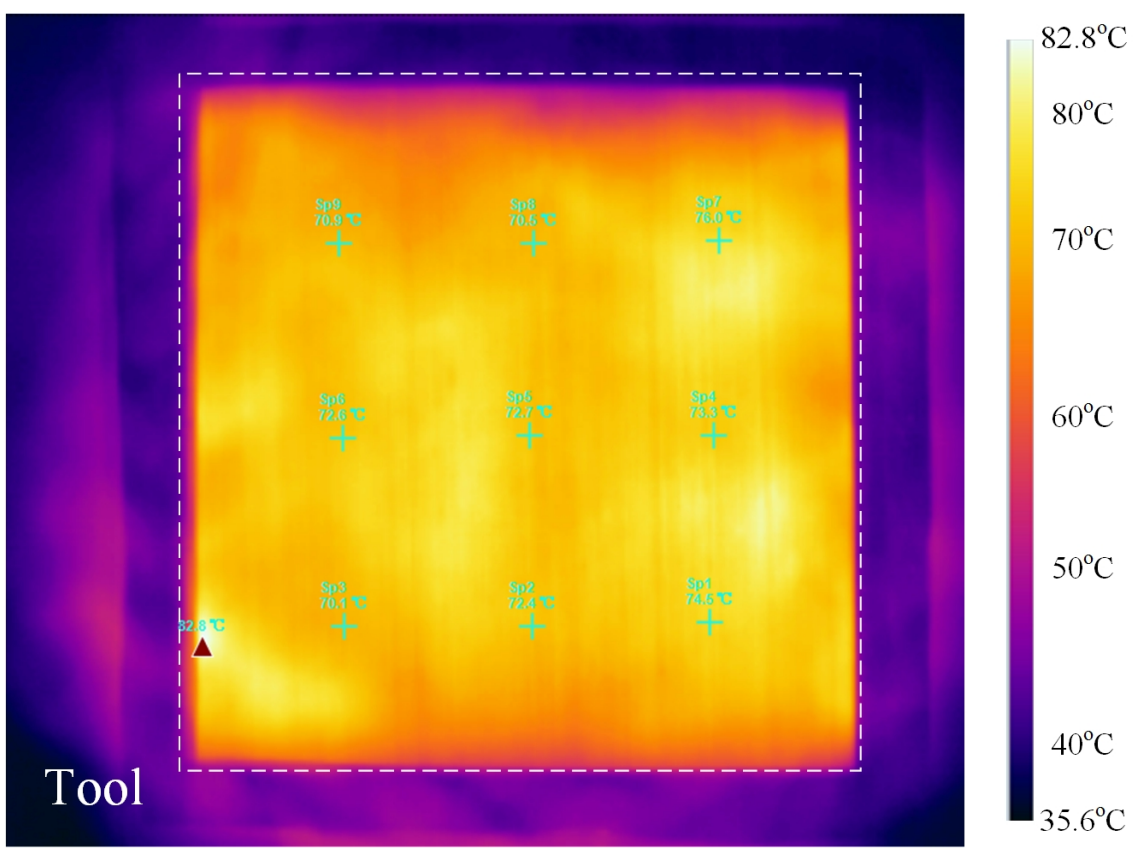

(b)

Multidirectional CFRP

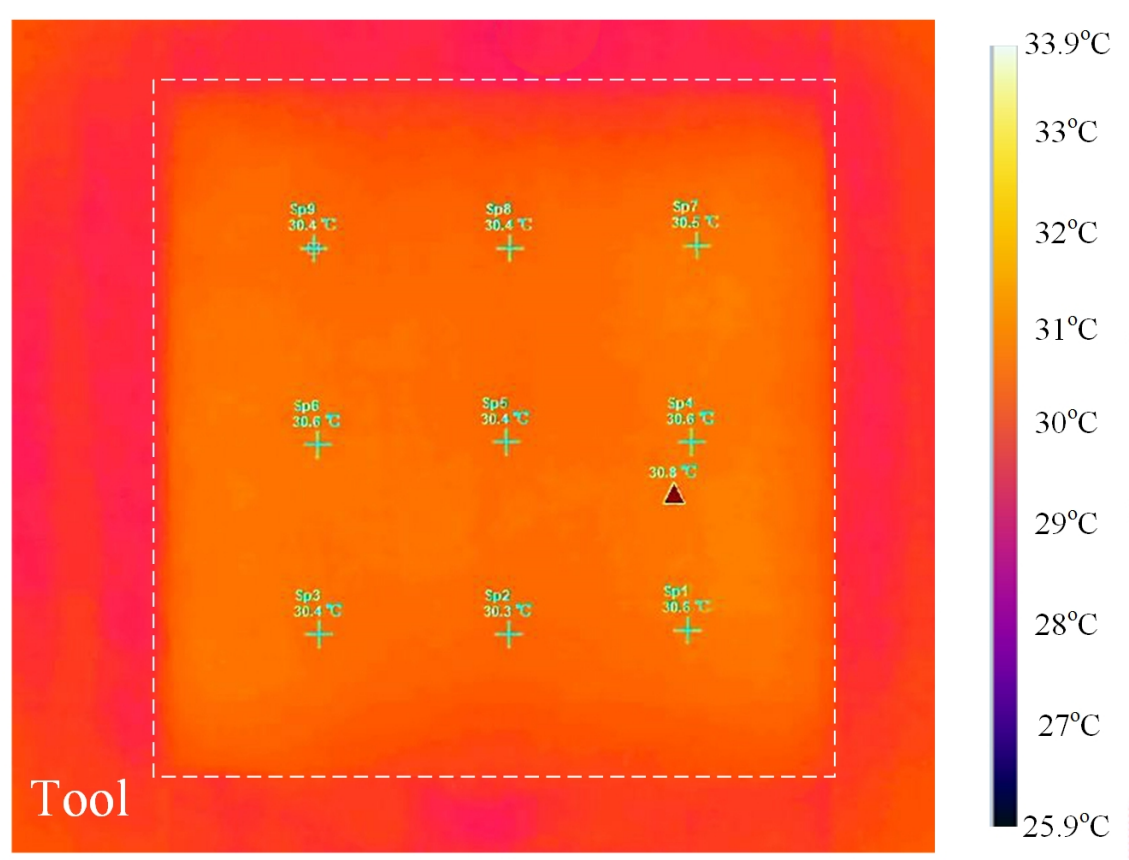




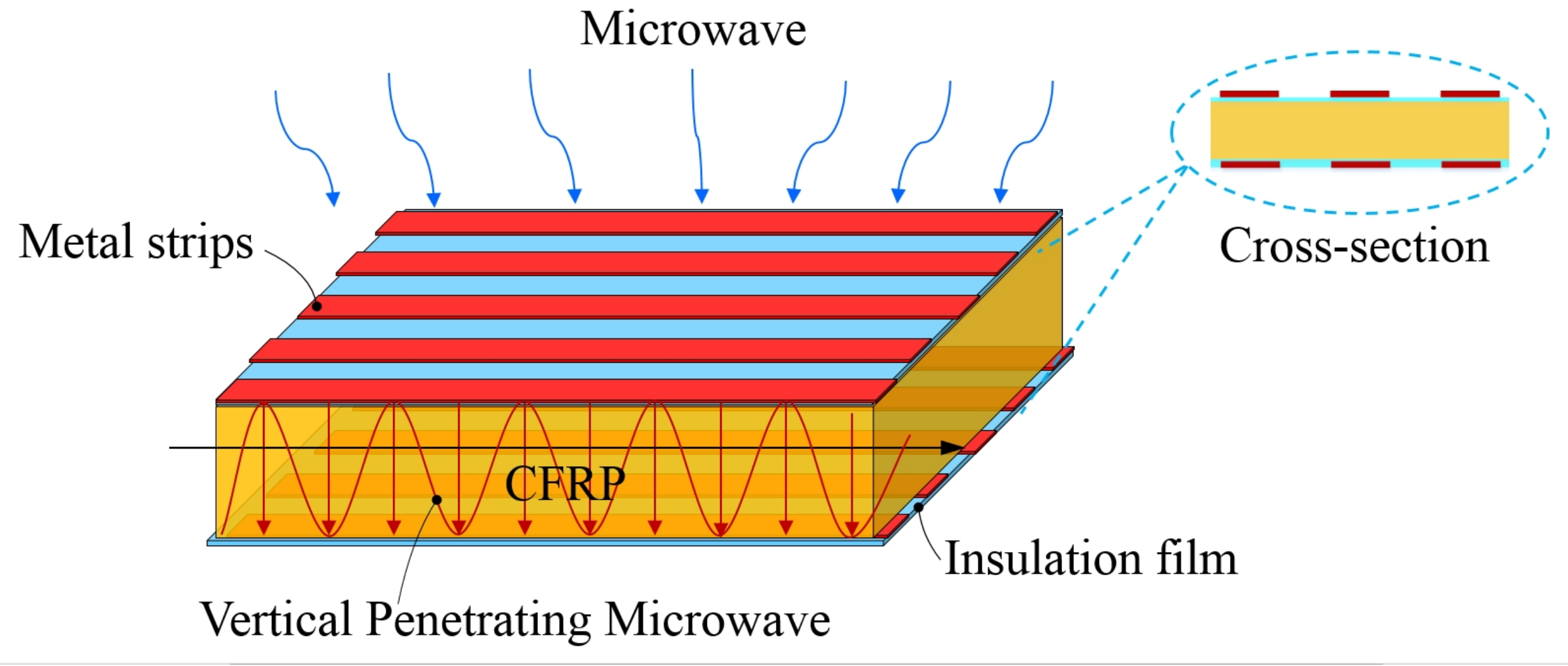



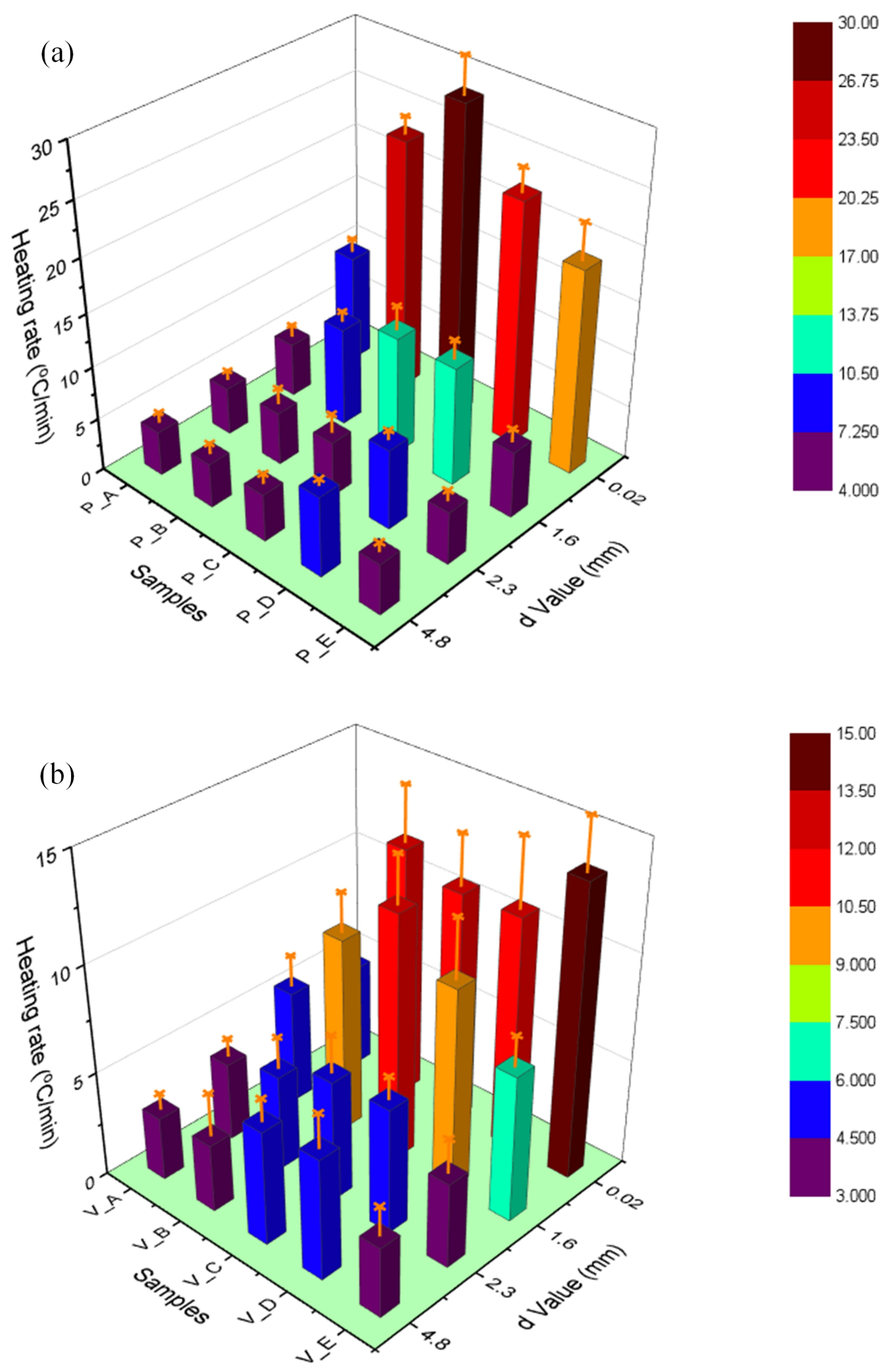


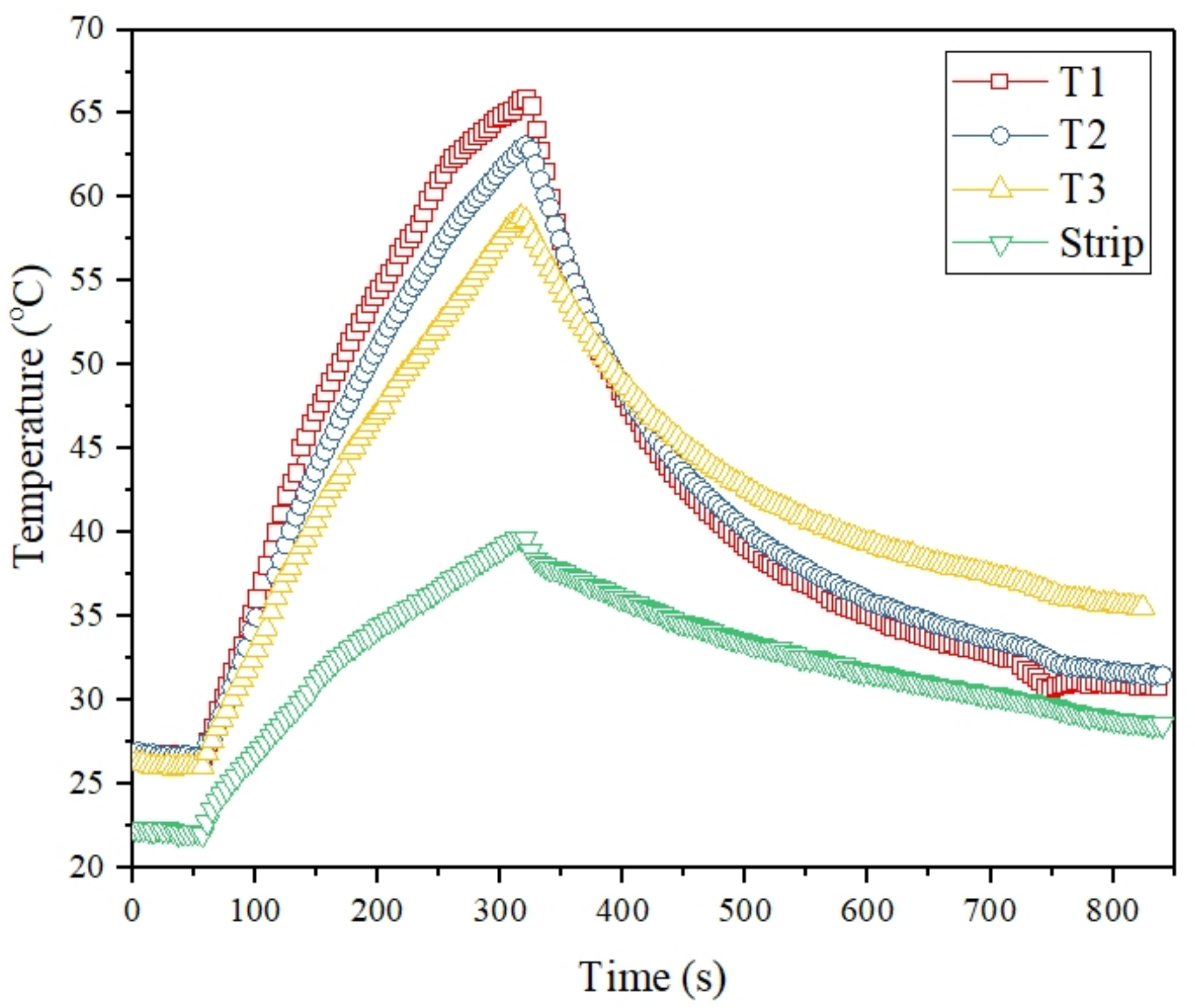


(a)

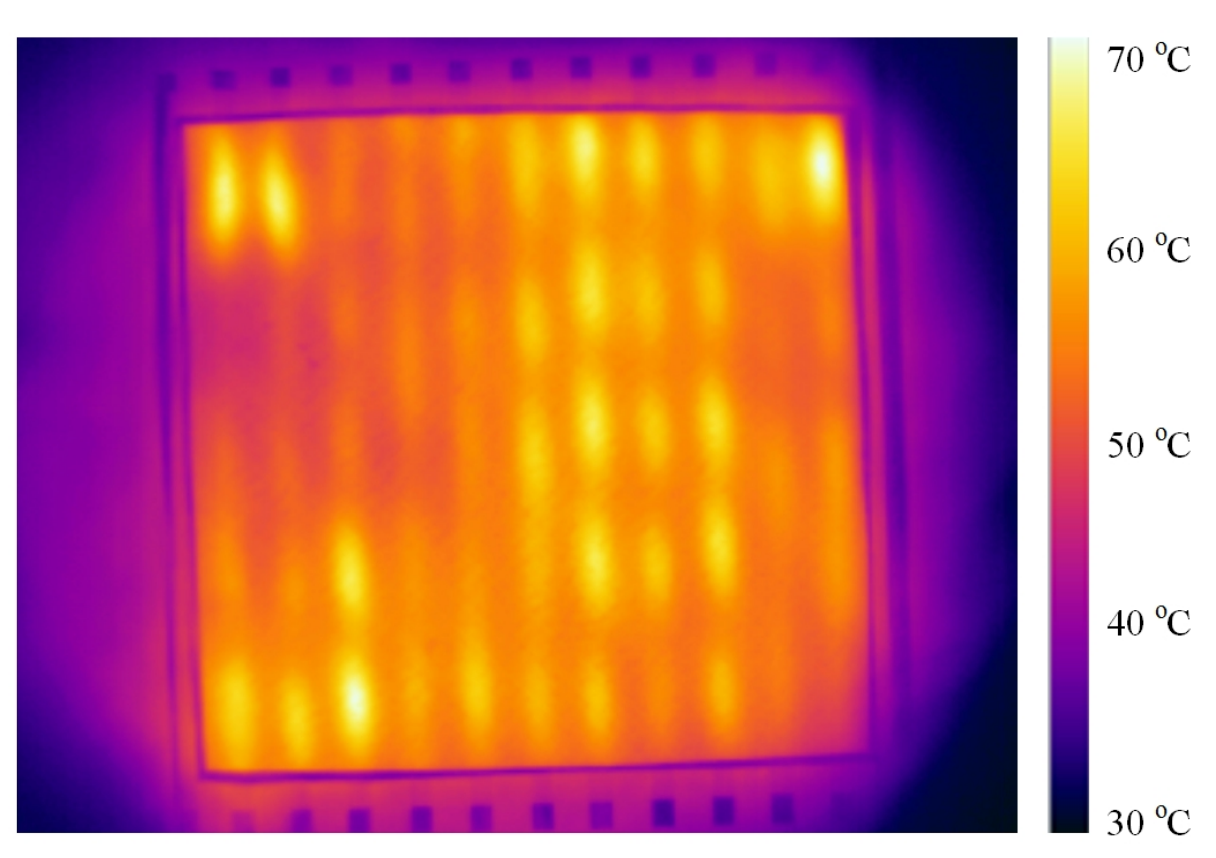

(b)

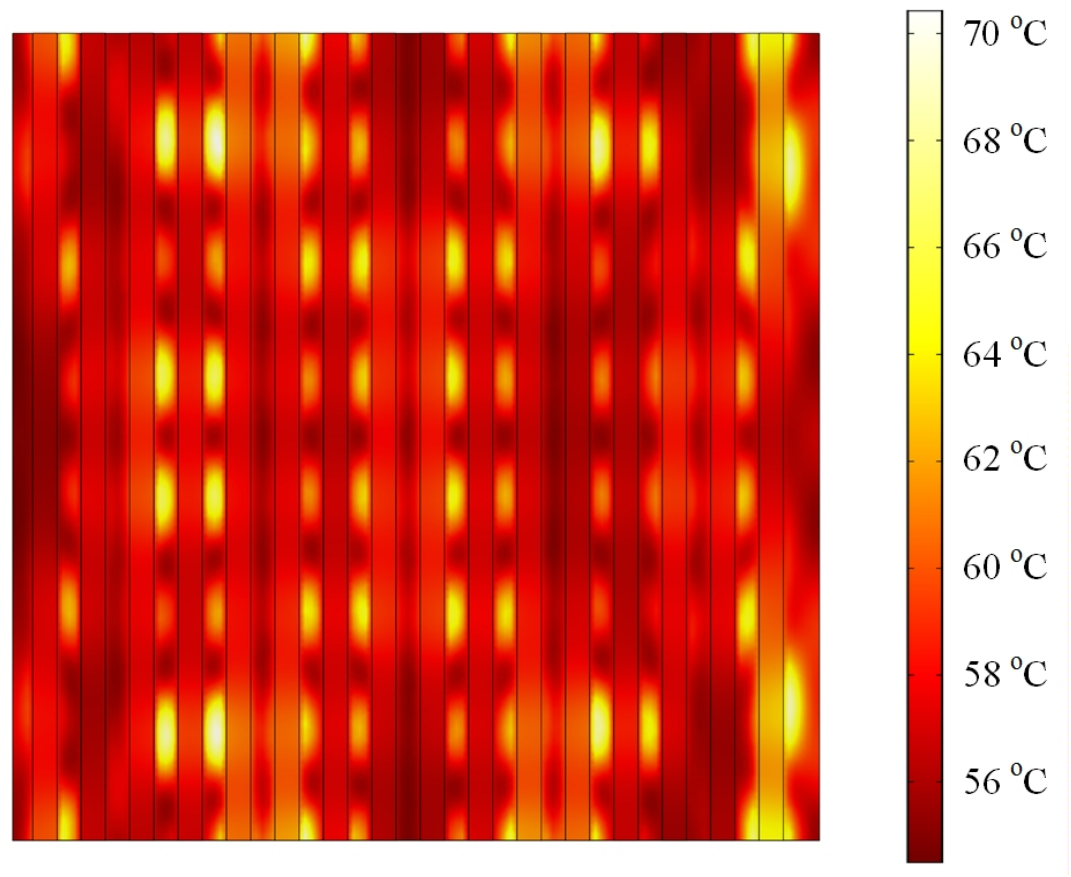


(a)

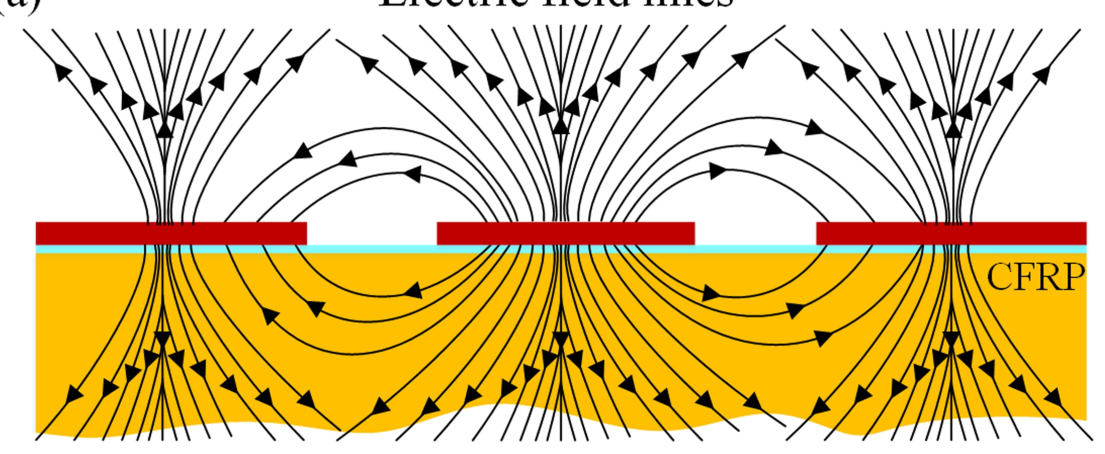

Odd mode

(c)

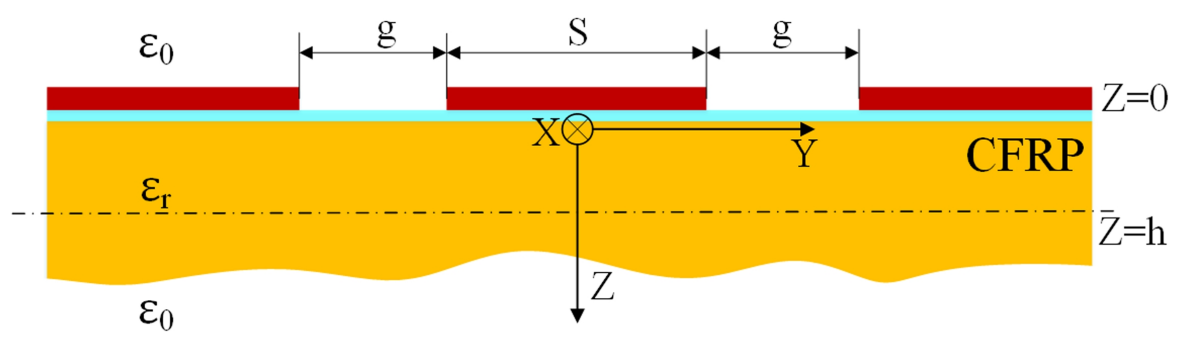

(b) Electric field lines



(d)

Electric wall

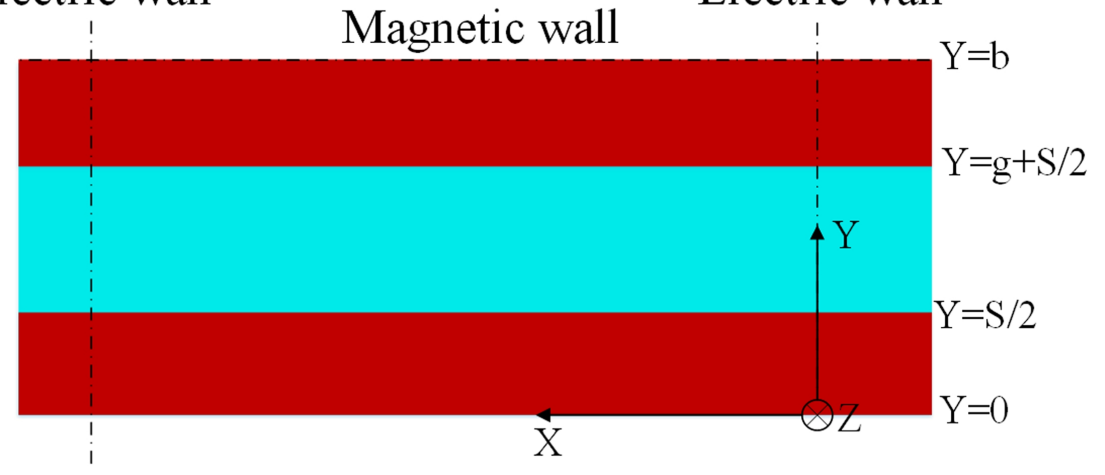


(a)

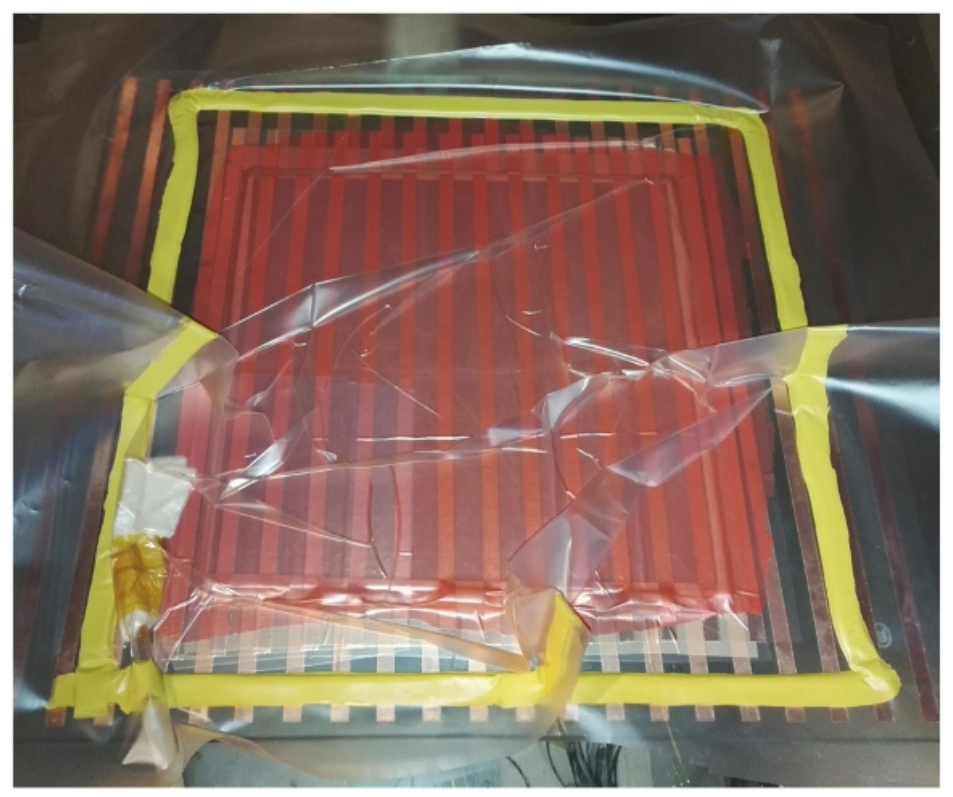

(c)

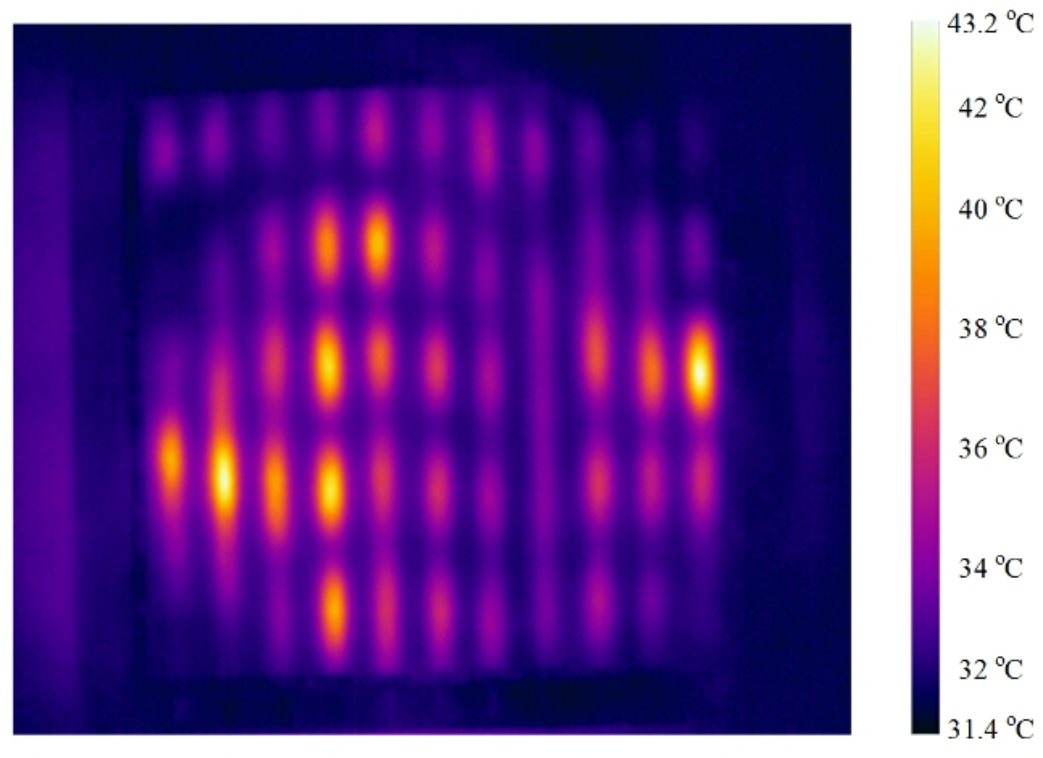

(b)

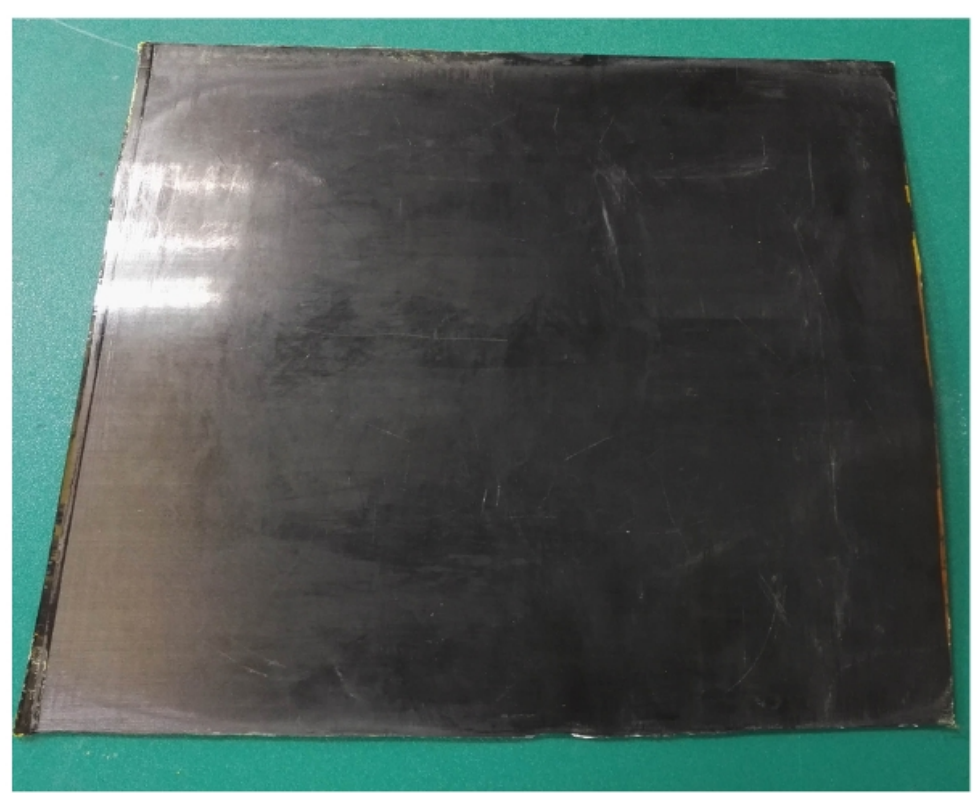

(d)

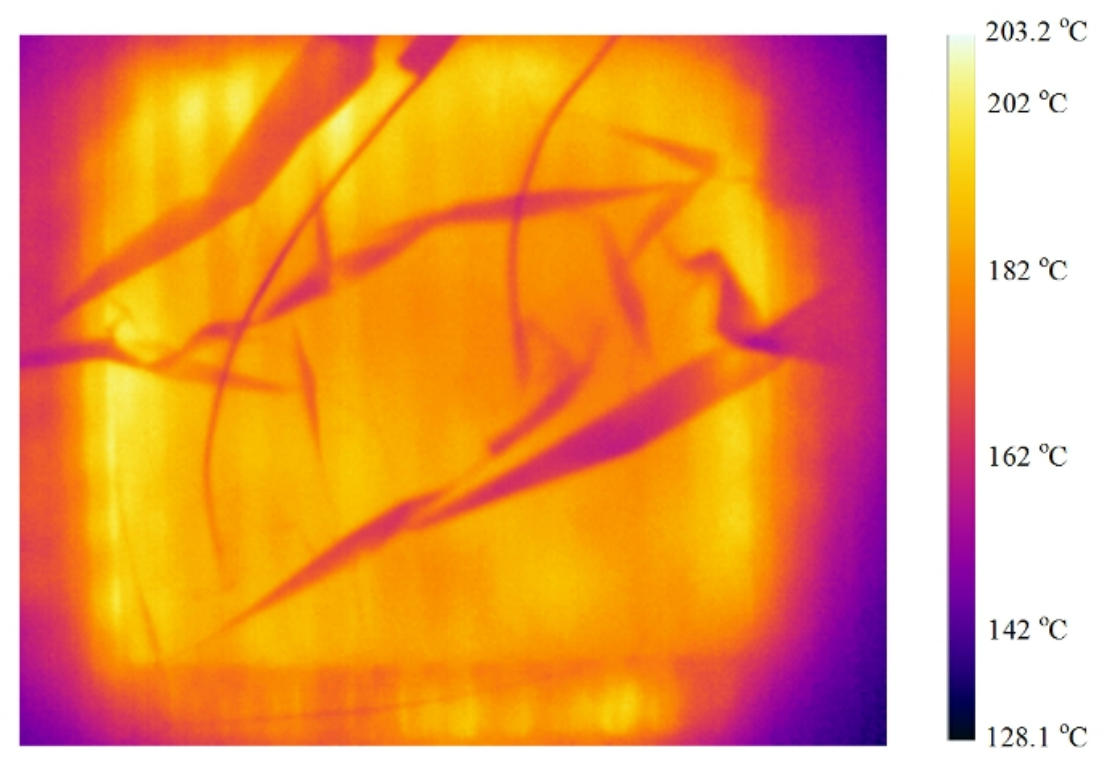




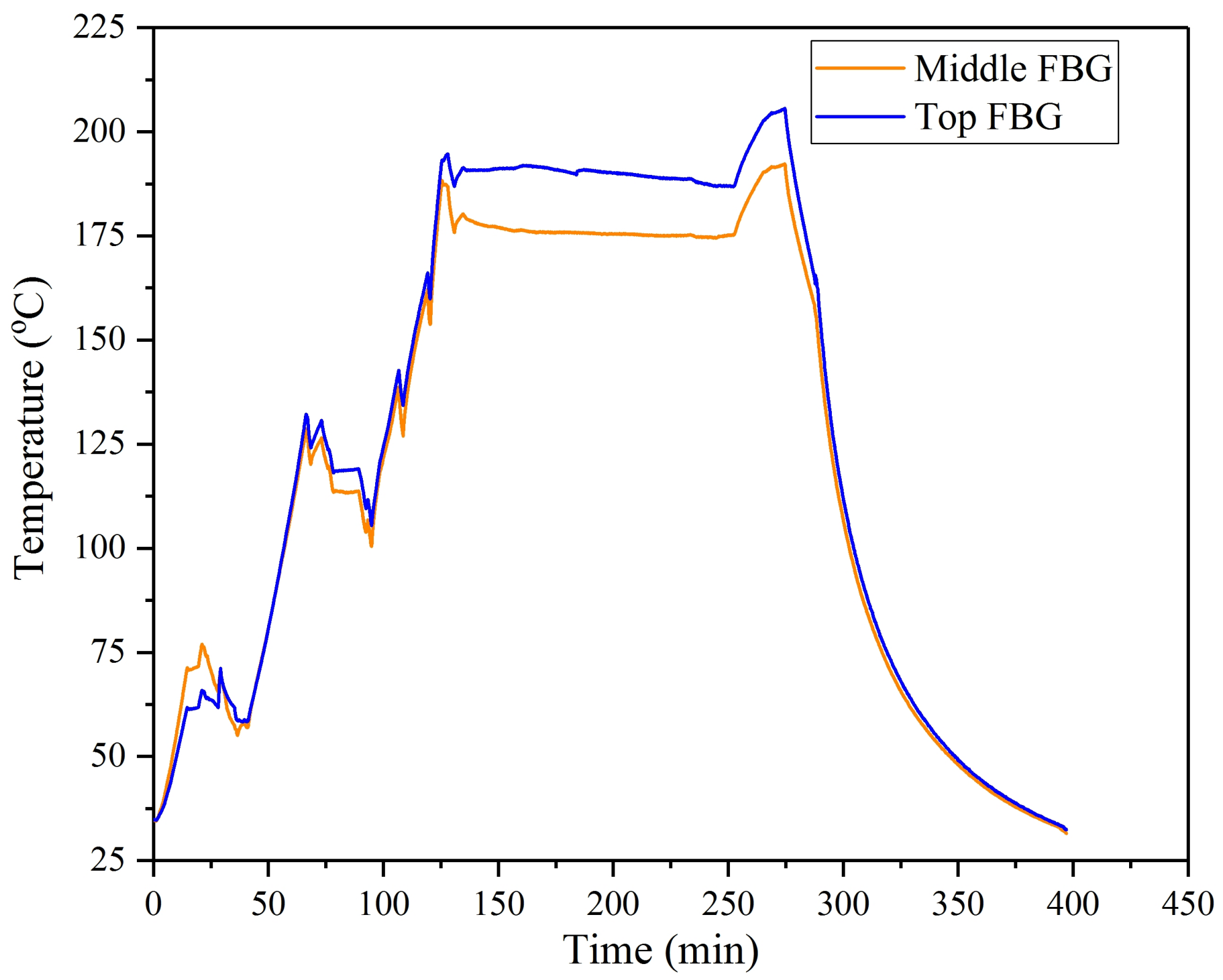


Table 1. Composite samples for microwave transmissivity testing

\begin{tabular}{cccc}
\hline & Thickness & Ply direction & Direction of electric field \\
\hline U_P_1 & $1 \mathrm{~mm}$ & {$\left[0^{\circ}\right]$} & Parallel to fiber axis \\
U_V_1 & $1 \mathrm{~mm}$ & {$\left[0^{\circ}\right]$} & Vertical to fiber axis \\
U_P_3 & $3 \mathrm{~mm}$ & {$\left[0^{\circ}\right]$} & Parallel to fiber axis \\
U_V_3 & $3 \mathrm{~mm}$ & {$\left[0^{\circ}\right]$} & Vertical to fiber axis \\
M_P_1 & $1 \mathrm{~mm}$ & {$\left[0^{\circ} /+45^{\circ} / 0^{\circ} / 45^{\circ} / 0^{\circ} /+45^{\circ} / 90^{\circ} /-45^{\circ}\right]$} & Parallel to fiber axis \\
M_V_1 & $1 \mathrm{~mm}$ & {$\left[0^{\circ} / 45^{\circ} / 0^{\circ} /-45^{\circ} / 0^{\circ} / 45^{\circ} / 90^{\circ} /-45^{\circ}\right]$} & Vertical to fiber axis \\
\hline
\end{tabular}

Table 2. Composite samples for the VPM heating rate testing

\begin{tabular}{cccc}
\hline Samples & $\begin{array}{c}\text { Width of copper } \\
\text { strip }\end{array}$ & $\begin{array}{c}\text { Width between } \\
\text { copper strips }\end{array}$ & $\begin{array}{c}\text { Height of insulation } \\
\text { material }\end{array}$ \\
\hline P_A & $4 \mathrm{~mm}$ & $5 \mathrm{~mm}$ & $0.02,1.6,2.3,4.8 \mathrm{~mm}$ \\
P_B & $6 \mathrm{~mm}$ & $9 \mathrm{~mm}$ & $0.02,1.6,2.3,4.8 \mathrm{~mm}$ \\
P_C & $8 \mathrm{~mm}$ & $13 \mathrm{~mm}$ & $0.02,1.6,2.3,4.8 \mathrm{~mm}$ \\
P_D & $10 \mathrm{~mm}$ & $17 \mathrm{~mm}$ & $0.02,1.6,2.3,4.8 \mathrm{~mm}$ \\
P_E & $12 \mathrm{~mm}$ & $21 \mathrm{~mm}$ & $0.02,1.6,2.3,4.8 \mathrm{~mm}$ \\
\hline
\end{tabular}

Table 3. Heating rates and ratios of the VPM at different thicknesses of the composite samples

\begin{tabular}{ccccc}
\hline Thickness $(\mathrm{mm})$ & 0 (Bottom) & 1.13 & 2.32 & 3.51 (Top) \\
\hline $\begin{array}{c}\text { VPM heating rate } \\
\left({ }^{\circ} \mathrm{C} / \text { min) }\right.\end{array}$ & 2.37 & 2.22 & 1.94 & 1.78 \\
$\begin{array}{c}\text { Thermal heating rate } \\
\left({ }^{\circ} \mathrm{C} / \mathrm{min}\right) \\
E_{\text {ratio }}\end{array}$ & 2.42 & 2.12 & 1.90 & 1.78 \\
\hline
\end{tabular}

